\title{
Structure and Composition of Isolated Core-Shell (In,Ga)N/GaN Rods Based on Nanofocus X-Ray Diffraction and Scanning Transmission Electron Microscopy
}

\author{
Thilo Krause, Michael Hanke, ${ }^{*}$ Lars Nicolai, Zongzhe Cheng, Michael Niehle, and Achim Trampert \\ Paul-Drude-Institut für Festkörperelektronik, Hausvogteiplatz 5-7, 10117 Berlin, Germany \\ Maik Kahnt, ${ }^{1,2}$ Gerald Falkenberg, ${ }^{1}$ and Christian G. Schroer ${ }^{1,2}$ \\ ${ }^{1}$ Deutsches Elektronen-Synchrotron (DESY), Notkestraße 85, 22607 Hamburg, Germany \\ ${ }^{2}$ Department Physik, Universität Hamburg, Luruper Chaussee 149, 22761 Hamburg, Germany \\ Jana Hartmann, ${ }^{3,4}$ Hao Zhou, ${ }^{3}$ Hergo-Heinrich Wehmann, ${ }^{3,4}$ and Andreas Waag ${ }^{3,4}$ \\ ${ }^{3}$ Institut für Halbleitertechnik and Laboratory for Emerging Nanometrology, \\ Technische Universität Braunschweig, 38092 Braunschweig, Germany \\ ${ }^{4}$ Epitaxy Competence Center ec ${ }^{2}$, Hans-Sommer-Straße 66, 38106 Braunschweig, Germany \\ (Received 16 November 2016; revised manuscript received 8 December 2016; published 28 February 2017)
}

\begin{abstract}
Nanofocus x-ray diffraction is used to investigate the structure and local strain field of an isolated (In, Ga) N/GaN core-shell microrod. Because the high spatial resolution of the x-ray beam is only $80 \times 90 \mathrm{~nm}^{2}$, we are able to investigate several distinct volumes on one individual side facet. Here, we find a drastic increase in thickness of the outer GaN shell along the rod height. Additionally, we performed highangle annular dark-field scanning-transmission-electron-microscopy measurements on several rods from the same sample showing that $(\mathrm{In}, \mathrm{Ga}) \mathrm{N}$ double-quantum-well and $\mathrm{GaN}$ barrier thicknesses also increase strongly along the height. Moreover, plastic relaxation is observed in the top part of the rod. Based on the experimentally obtained structural parameters, we simulate the strain-induced deformation using the finiteelement method, which serves as the input for subsequent kinematic scattering simulations. The simulations reveal a significant increase of elastic in-plane relaxation along the rod height. However, at a certain height, the occurrence of plastic relaxation yields a decrease of the elastic strain. Because of the experimentally obtained structural input for the finite-element simulations, we can exclude unknown structural influences on the strain distribution, and we are able to translate the elastic relaxation into an indium concentration which increases by a factor of 4 from the bottom to the height where plastic relaxation occurs.
\end{abstract}

DOI: 10.1103/PhysRevApplied.7.024033

\section{INTRODUCTION}

The incorporation of indium atoms into a GaN matrix offers the possibility of tuning the emitted wavelength through the full optical spectrum [1-3]. This quality makes the ternary (In, Ga)N alloy embedded as quantum-well structures into a GaN matrix interesting for a variety of optoelectronic applications. To improve and expand existing group-III-nitride-based semiconductor technologies-which nowadays are mainly based on planar systems - researchers have focused their activities on the investigation of lowdimensional, mesoscopic structures such as quantum dots and nanowires $[4,5]$. For example, $(\mathrm{In}, \mathrm{Ga}) \mathrm{N} / \mathrm{GaN}$ coreshell nanowires and rods are discussed as promising candidates for next-generation light-emitting diodes [6-11]. If the structures are grown not in an axial but in a coreshell geometry, the optically active area can be significantly increased compared to planar structures $[12,13]$. Moreover, concerning $\mathrm{GaN}$, the growth of ( $\mathrm{In}, \mathrm{Ga}) \mathrm{N}$ quantum wells on

\footnotetext{
*hanke@pdi-berlin.de
}

nonpolar $m$ planes is especially interesting because the optical performance is not influenced by polarization potentials or the quantum-confined Stark effect $[14,15]$. Hence, indium content and quantum-well width mainly determine the band gap.

However, in contrast to planar structures, the structural analysis and the determination of the chemical composition of low-dimensional objects requires new characterization approaches with a focus on spatial resolution, such that fluctuations in the submicrometer regime can be detected. An important question to address is the homogeneity within large ensembles - but also of individual ensemble members themselves. Here, the analysis focus lies on the structural quality, e.g., the defects [16], the crystal phases [17-19], the interface quality, and the overall structural condition of complicated heterostructure objects [20-23].

A challenging ongoing task remains the determination of the indium content in low-dimensional (In, Ga)N-based heterostructures. Here, the situation becomes more complicated due to related phenomena such as indium clustering and segregation [24-26]. As these objects are truly three 
dimensional, tomographic investigations have also been pursued [27], and, obviously, the analysis of the strain field, which is often more complex than in the planar case, is an important task [28-30] not only because the strain might be used to directly engineer the band gap [31,32].

Compared to other methods, nanofocus x-ray diffraction becomes an increasingly popular approach to investigate local strain fields in low-dimensional heterostructure semiconductors [33-37]. Owing to the focus sizes in the submicrometer range, nanofocus $\mathrm{x}$-ray diffraction proves to be an ideal method for analyzing $\mu \mathrm{m}$-sized and smaller structures in terms of strain and structure [38-41]. For example, using scanning x-ray-diffraction microscopy, full strain maps under a certain Bragg condition can be obtained for individual rods [42] or arrays [43]. The high sensitivity of the $\mathrm{x}$-ray beam with respect to the strain allows us to draw instructive conclusions about the structural and chemical constitution. This information can then be used to understand and improve the sample fabrication and performance.

This paper is organized as follows. In Sec. II, we describe the growth process of the core-shell ( $\mathrm{In}, \mathrm{Ga}) \mathrm{N} / \mathrm{GaN}$ rods and the focused-ion-beam sample preparation. Section III introduces the experiments performed for analyzing the structural constitution and chemical composition with spatial resolution. Therefore, we perform nanofocus x-raydiffraction experiments complemented by nanofocus x-ray reflectivity, as described in Sec. III A. We interpret the results by comparing the experimental data to kinematic scattering simulations based on finite-element simulations of the strain-induced deformation field. Furthermore, results from scanning-transmission-electron-microscopy measurements which are performed on a series of rods from the very same sample are shown in Sec. III B. Finally, we summarize the paper in Sec. IV.

\section{SAMPLE GROWTH AND FOCUSED-ION-BEAM SAMPLE PREPARATION}

The (In, Ga)N/GaN core-shell microrods comprise an $n$-doped GaN core surrounded by two 5 -nm-thick (In, Ga)N quantum wells (QWs) with an intended indium incorporation of about $30 \%$. In between the double quantum well (DQW), a GaN barrier of $5 \mathrm{~nm}$ is grown. A $p$-doped $\mathrm{GaN}$ shell of $100 \mathrm{~nm}$ surrounds the DQW. The rods are grown by selective-area metalorganic vapor phase epitaxy in a closecoupled showerhead reactor under the continuous growth mode. The template consists of a 30-nm-thick $\mathrm{SiO}_{x}$ mask on a $\mathrm{GaN}$ buffer layer on [0001]-oriented sapphire $\left(\mathrm{Al}_{2} \mathrm{O}_{3}\right)$. Holes in the dielectric mask are produced by photolithography and reactive-ion etching by inductively coupled plasma. These holes have diameters of $800 \mathrm{~nm}$ defining the width of the GaN core and are arranged in a hexagonal pattern with a pitch of $2.4 \mu \mathrm{m}$ determining the rod distribution as shown in Fig. 1(a). The template is heated up in the reactor under $\mathrm{N}_{2}$ carrier gas before a short growth step under a high V/III ratio of 1000 and under $\mathrm{H}_{2}$ carrier

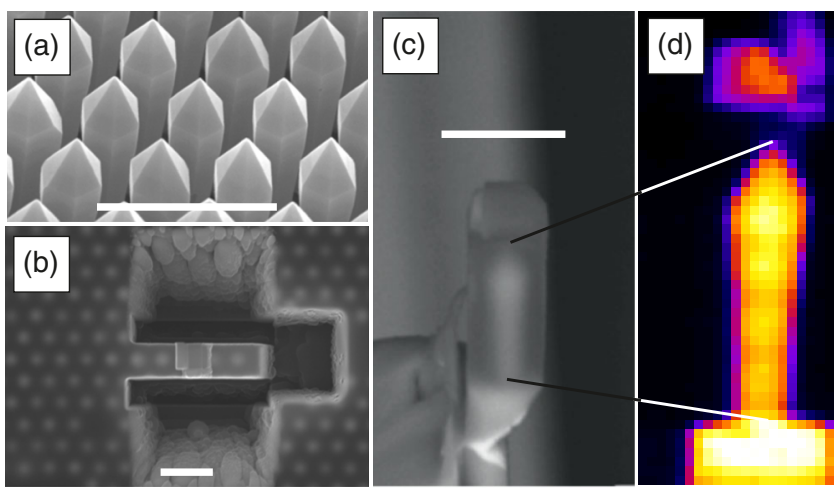

FIG. 1. (a) Scanning electron micrograph of the as-grown (In, Ga)N/GaN core-shell microrods. Before focused-ion-beam preparation, the rods are embedded in polymer glue. To isolate a lamella, trenches are cut as shown in (b). (c) Finally, the isolated rod is mounted on a $\mathrm{Cu}$ grid. (d) The $\mathrm{Ga} K-\alpha_{1}$ fluorescence is shown on a map recorded by scanning the nanofocus $\mathrm{X}$-ray beam over the sample. On top of the glue (about $2 \mu \mathrm{m}$ above the rod) a Ga droplet resulting from focused-ion-beam preparation can be observed. The white scale bar refers to a length of $5 \mu \mathrm{m}$.

gas is performed to start the growth of the GaN cores in the hole openings. Subsequently, the 3D-growth step of the microrods is carried out under a low V/III ratio of 77 and a $\mathrm{SiH}_{4}$ flow of $37 \mathrm{nmol} / \mathrm{min}$ for an $n$-type doping and an increased vertical growth rate $[44,45]$. The temperature during this 20-min-long step is held at $1060^{\circ} \mathrm{C}$. The shell growth steps are all conducted at a high $\mathrm{V} / \mathrm{III}$ ratio again: starting with a low- and high-temperature $\mathrm{GaN}$ spacer layer, continuing with two QWs embedded in a GaN barrier, and terminating with several Mg-doped $\mathrm{GaN}$ layers for $p$-type doping. The QWs are grown at a real temperature of $690^{\circ} \mathrm{C}$. An in situ annealing is performed for $10 \mathrm{~min}$ at $770^{\circ} \mathrm{C}$ after the growth in order to activate the $\mathrm{Mg}$ dopants.

Using a focused $\mathrm{Ga}^{+}$ion beam (FIB), we extracted a single rod from the sample. To protect the rod from Ga implantation, the sample is embedded in epoxy glue diluted with acetone to reduce the viscosity. With a carbon depot on top, the conductivity is increased such that the charging of the sample is alleviated. In a first step, trenches are cut next to the lamella, as seen in the scanning electron micrograph (SEM) shown in Fig. 1(b). With a manipulator needle, the lamella containing several rods is lifted out and mounted on a copper grid commonly used for transmission-electron-microscopy experiments. In a series of fine-polishing steps, the investigated rod shown in Fig. 1(c) is isolated and cleaned from redeposited $\mathrm{GaN}$ crystallites [46]. The FIB preparation ensures that the detected $\mathrm{x}$-ray scattering stems only from the single object and that it is not superimposed by potential neighbors.

\section{EXPERIMENTS AND DISCUSSION}

\section{A. X-ray diffraction with sub-100-nm spatial resolution}

To investigate the local strain field in nanometer-sized structures-as, here, the (In,Ga)N DQW-one usually 
relies on highly specialized nanofocus x-ray-diffraction setups available at third-generation synchrotrons. The presented data are recorded at the nanoprobe beamline P06 at PETRAIII at DESY in Hamburg, Germany [47], where a focus size of $80 \times 90 \mathrm{~nm}^{2}$ is reached using nanofocusing refractive $x$-ray lenses $[48,49]$. With an additional prefocusing lens, a photon flux of about $10^{9}$ photons/sec is generated at a photon energy of $17 \mathrm{keV}$. All x-ray data are recorded using a Pilatus $300 \mathrm{~K} 2 \mathrm{D}$-area detector. In a first step, we used an optical microscope to align the sample in the focus point of the x-ray beam which we found via a ptychographic reconstruction of a Siemens star. For a more accurate positioning of the beam on the rod facet, we employ $\mathrm{x}$-ray fluorescence. $\mathrm{A} \mathrm{Ga} \mathrm{K}-\alpha_{1}$ fluorescence map of the rod is shown in Fig. 1(d). Owing to the fact that the outer facet is not perpendicular to the substrate but rather is inclined, we have to realign the sample with respect to the $\mathrm{x}$-ray beam for each position individually.

We measure the in-plane strain field normal to the $m$-plane facet which is the hexagonal $\langle 1 \overline{1} 00\rangle$ direction using nanofocus $\mathrm{x}$-ray diffraction. The measurements are performed at four positions on one individual facet of the isolated rod, as sketched in Figs. 2(a)-2(d). The distance between the positions is about $1 \mu \mathrm{m}$. All data shown in the following are labeled accordingly such that they can be directly identified with these four heights. At these positions, we perform rocking curves over $4^{\circ}$ around the $\mathrm{GaN}$ (1100) Bragg reflection with an angular resolution of $0.01^{\circ}$. For each scan series, we chose a region of interest around the Bragg reflection on the detector frame and integrated over approximately $0.001 \AA^{-1}$ in the vertical direction. Based on these line scans, we are able to reconstruct a $2 \mathrm{D}$ slice in reciprocal space which is defined by the scattering plane and the facet normal. At each height, the scans are

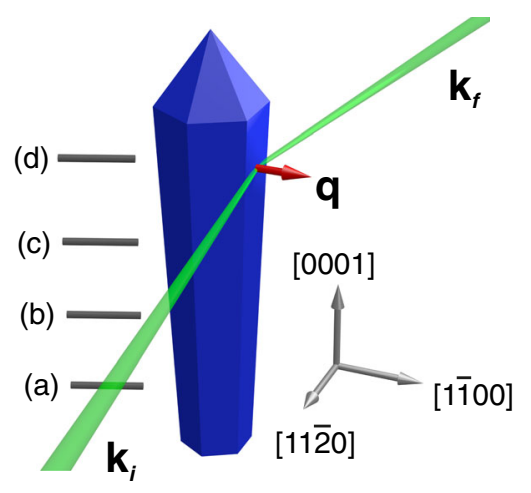

FIG. 2. Sketch of the applied diffraction geometry. To investigate the strain field normal to the $m$-plane facet, the hexagonal (1100) reflection is measured. The diffraction plane lies parallel to the substrate surface and is spanned by the incoming beam $\mathbf{k}_{i}$ and the diffracted beam $\mathbf{k}_{f}$, defining the scattering vector $\mathbf{q}=\mathbf{k}_{f}-\mathbf{k}_{i}$. The measurements are performed at four heights, labeled (a), (b), (c), and (d). These position labels are referred to in the following presented data. repeated at several horizontal positions to ensure that we have actually hit a position directly on the facet.

Figure 3 shows the diffusely scattered $x$-ray-diffraction intensity in reciprocal space around the GaN (1100) reflection. In the radial direction $\left(q_{\mathrm{rad}}\right)$, the $(1 \overline{1} 00)$ reflection is sensitive to the in-plane strain normal to the $m$-plane facet. Hence, it directly measures the in-plane deformation of the $\{1 \overline{1} 00\}$ lattice planes induced by the lattice mismatch between the (In, Ga)N DQW and the surrounding GaN matrix. Moreover, the diffraction profile along $q_{\text {rad }}$ offers a way to quantify the indium content incorporated in the DQW.

In the angular direction ( $\left.q_{\text {ang }}\right)$, the reflection is sensitive to the hexagonal rod geometry. The rod's shape function shows a six-armed star and, along each of the six crystal truncation rods (CTRs), oscillations are visible containing direct information about the structural constitution of the illuminated volume. The fact that up to nine oscillations are visible, e.g., in Fig. 3(a), originates from the high crystal quality of the illuminated volume together with the small focus of the $\mathrm{x}$-ray beam corresponding to the averaged information of a comparatively small volume. Such details can be resolved in ensemble measurements only if all of the scattering objects are perfectly identical [50].

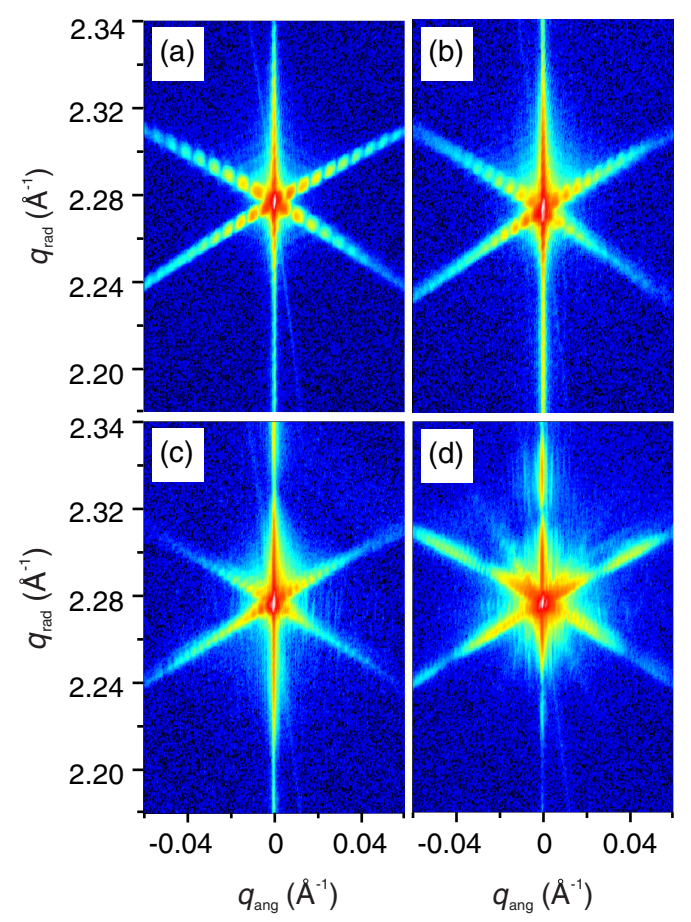

FIG. 3. Reciprocal space maps of the diffusely scattered logarithmic intensity around the GaN (1100) reflection. The maps are recorded at four positions (a) to (d) on one individual facet, as sketched in Fig. 2. Along the angular direction, the shape function of the rod is visible in the form of a six-armed star. The crystal truncation rods are modulated by the shape function related to the structural constitution of the illuminated volume. The radial direction is sensitive to the strain induced by the two (In, Ga)N quantum wells. 
Figures 3(a)-3(d) correspond to the rod heights sketched in Fig. 2. Obviously, the shape functions for all four heights look very different, indicating a strong structural inhomogeneity along the height of the investigated facet. For example, it is obvious that the periodicity along the CTRs increases with an increasing height on the rod. These Kiessig fringes are the Fourier transform of the shell sequence in the facet. In fact, the retrieved thickness values $\left(d_{\text {shell }}=2 \pi / \Delta q\right)$ show that these fringes are related to the thickness of the outer GaN shell surrounding the DQW. Interestingly, we find an increase in thickness towards the tip by a factor of 3 . Moreover, Fig. 3(d) shows larger oscillations modulating the CTRs which refer to a thickness of about $16 \mathrm{~nm}$. A comparison to scanning-transmission-electron-microscopy (STEM) measurements presented in Fig. 7 indicates that these oscillations are related to the GaN barrier. All structural parameters retrieved from $\mathrm{x}$-ray-diffraction experiments are shown in blue in Table I and serve as structural input for subsequent finite-element method (FEM) simulations.

Based on the structural parameters shown in Table I, we simulate the strain-induced linear-elastic deformation inherent to the rod for positions (a)-(d) employing the FEM as implemented in the commercial software package MARC MENTAT $^{\circledR}$. The values for the DQW and GaN barrier thicknesses for models (a), (b), and (c) are geared to the STEM measurements given in Fig. 7. As these values are in the range of only a few nanometers, they result in comparatively large oscillations along the CTRs in x-raydiffraction patterns. Because of the applied scan range, it

TABLE I. Structural parameters and indium content used to model the deformation field within a finite-element approach. The parameters in blue are retrieved from nanofocus $\mathrm{x}$-ray diffraction (XRD). The facet widths written in red are obtained from X-ray-reflectivity (XRR) experiments (see Fig. 5). The other structural parameters shown in black are geared to the STEM measurements presented in Sec. III B. These values are taken at positions where the identical GaN shell thickness is measured (see Fig. 2). The indium content is retrieved by fitting the kinematic scattering simulations to experimental data shown in Fig. 4. The sketch illustrates the respective structural parameters and the method by which they are obtained.

\begin{tabular}{|l|c|c|c|c|}
\hline \multicolumn{5}{|c|}{ FEM simulation parameters } \\
\hline Position & (a) & (b) & (c) & (d) \\
\hline GaN shell (nm) & 90 & 110 & 170 & 270 \\
\hline GaN barrier (nm) & 4 & 6 & 6 & 16 \\
\hline (In,Ga)N QWs (nm) & 4 & 4 & 5 & 9 \\
\hline Facet width (nm) & 650 & 700 & 750 & 850 \\
\hline \hline In content (\%) & 6 & 10 & 22 & 18 \\
\hline
\end{tabular}

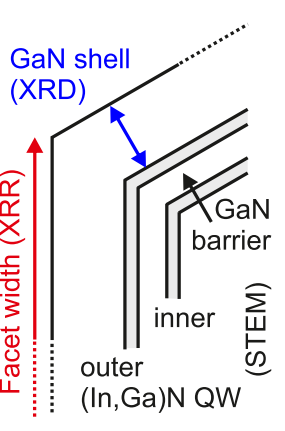

is not possible to resolve these oscillations in the presented data (see Fig. 3). Additionally, we implement different facet widths for all four positions, which are shown in red in Table I. These values are deduced from the nanofocus X-rayreflectivity experiments presented in Fig. 5, which are performed at the very same heights as sketched in Fig. 2. For modeling the mechanical properties of the materials, we use the elastic constants for $\mathrm{GaN}$ and $\mathrm{InN}$ given in Ref. [51] and interpolate the ones for the $\operatorname{In}_{x} \mathrm{Ga}_{1-x} \mathrm{~N}$ alloy linearly (where $x$ is the indium content).

The retrieved deformation field induced by DQW, $\mathbf{u}$, is used to compute the displacement $\mathbf{u}(\mathbf{r})$ of the atomic positions $\mathbf{r}$ of the wurtzite $\mathrm{GaN}$ crystal. The atomic coordinates and their displacement enter the subsequent kinematic scattering simulations which we apply to compute the scattered intensity in reciprocal space around the $\mathrm{GaN}$ (1100) reflection. In order to mimic the situation of the nanofocus x-ray-diffraction experiment, we append a Gaussian beam profile to the kinematic sum

$$
I(\boldsymbol{q}) \propto\left|\sum_{i} \frac{e^{-\left(r_{i, x}-\mu\right)^{2} / 2 \sigma^{2}} e^{i \boldsymbol{q} \cdot\left(\boldsymbol{r}_{i}+\boldsymbol{u}\left(\boldsymbol{r}_{i}\right)\right)}}{\sqrt{2 \pi} \sigma}\right|^{2},
$$

such that we can define the beam position $(\mu)$ and the FWHM, which is related to the variance via $\sigma=$ FWHM $/ 2 \ln (2)$ [41]. Hence, we are able to compute the scattered intensity $I$ as a function of the scattering vector $\mathbf{q}$ originating from a distinct volume of the rod. Because of the comparatively small size of the illuminated rod volume, it is justified to neglect multiple scattering events of a single photon such that the kinematic plane-wave approach remains valid [34].

Figure 4 displays line scans along $q_{\mathrm{rad}}$ around the GaN (1100) reflection. For each height, a simulated line profile (top panels) based on the FEM and the according experimental scan (bottom panels) extracted from Fig. 3 are shown. All experimental line profiles look very characteristic and show distinct features of the illuminated volume. The FEM simulations suggest that most parts of the wire constitute relaxed GaN. Consequently, all line profiles show a strong peak at the expected position of relaxed GaN at $q_{\mathrm{rad}}=2.277 \AA^{-1}$. Furthermore, fine oscillations which modulate the line profiles refer to the shape function of the outer $\mathrm{GaN}$ shell and reveal a clear trend towards higher periodicity from Fig. 4(a) to Fig. 4(c), indicating an increase of the outer $\mathrm{GaN}$ shell thickness. Underlying the fine oscillations, broader oscillations are visible whose frequency also increases with an increasing height position on the rod.

The simulated profiles are computed using kinematic scattering theory applying Eq. (1), where we assume a beam width of $90 \mathrm{~nm}$ and, in all cases, we position the beam maximum in the middle of the DQW. The displacement field of the atomic positions serves as the input for the scattering simulation, which is obtained via interpolation with the 


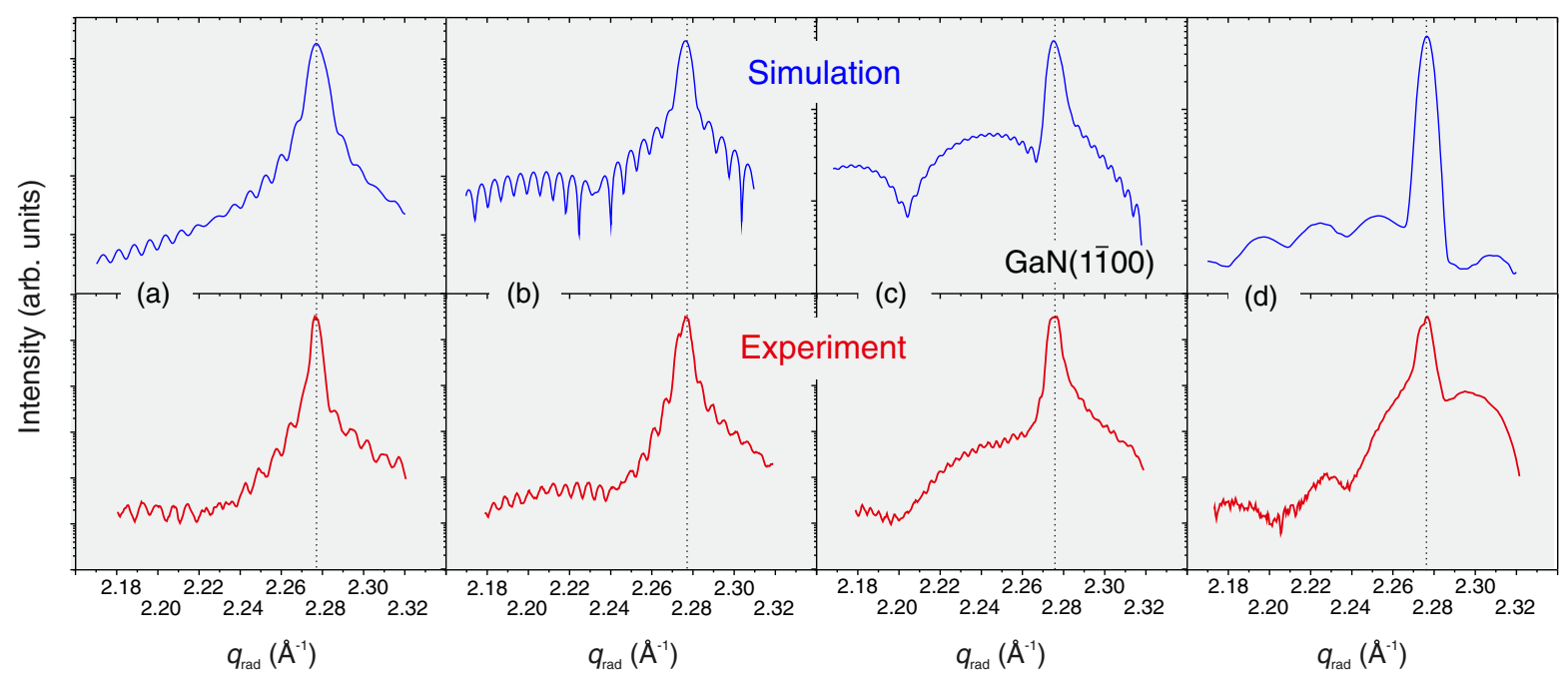

FIG. 4. (Top panels) Simulated and (bottom panels) experimentally measured line scans along $q_{\text {rad }}$ of the logarithmic intensity through the GaN (1100) reflection. The columns, labeled (a), (b), (c), and (d), each refer to one height position on the rod; see Fig. 2. The experimental line scans are extracted from Fig. 3. The simulated line scans are computed using kinematic scattering theory based on the deformation field retrieved from finite-element calculations using the parameters given in Table I as input.

displacement field computed with the FEM. Hence, the parameters used for the FEM simulation (see Table I) can be used to fit the simulated line profile to the experimental data.

The broader oscillations are sensitive to the superpositions of the GaN barrier and the DQW which define the distance between the two minima. The indium content mainly shifts the minima position along $q_{\mathrm{rad}}$. At positions (a) and (b), the GaN barrier and DQW thicknesses have a stronger impact on the peak shape, and the indium content mainly changes the amplitude of the oscillations. In cases (c) and (d), the variation of the indium content has a greater impact on the position of the minima. While the simulations describe the experimental data very accurately in (a) and (b),

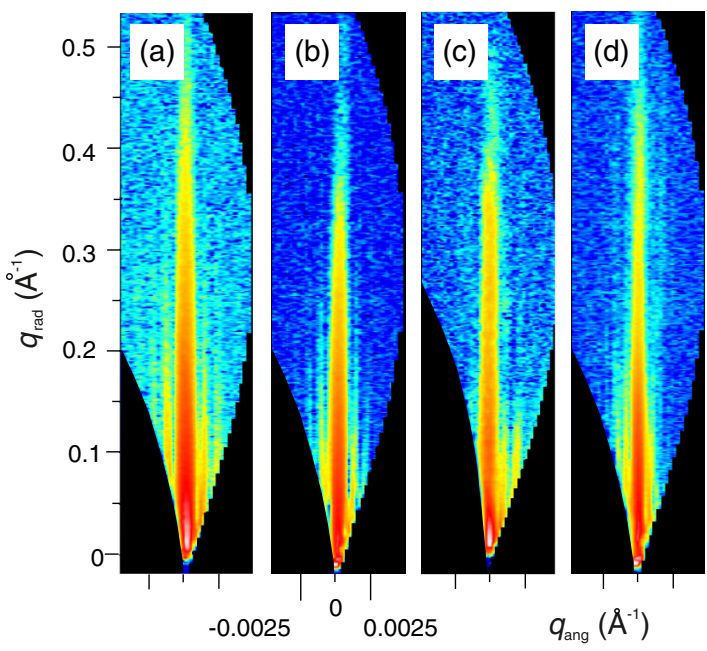

FIG. 5. Reciprocal space maps of the reflected intensity in a logarithmic scale. (a), (b), (c), and (d) refer to the heights sketched in Fig. 2. In the $q_{\text {ang }}$ direction, sattelites next to the CTR are visible whose periodicity refers to the facet width. there is an increasing discrepancy visible in (c) and (d). We attribute this observation to the presence of structural inhomogeneities, as, e.g., the onset of plastic relaxation towards the top. Concerning the sensitivity of the simulated line profiles, it is worth mentioning that a variation of the indium content of $\pm 1 \%$, as well as a thickness variation of $\pm 1 \mathrm{~nm}$ of the quantum well or the barrier has a clearly visible impact. Moreover, it is important to note that the better we know the structural constitution of the rod, the more accurately we are able to determine the indium content via FEM simulations-which, of course, demand that relaxation occurs purely elastically.

From STEM measurements (see Sec. III B) performed on a representative rod from the same sample, we are able to estimate the $\mathrm{GaN}$ barrier and quantum-well thicknesses shown in black in Table I. Hence, the only free remaining parameter in the FEM simulations is the indium content. Consequently, within the framework of linear-elasticity theory, we can determine the indium content with comparatively high accuracy. The obtained values for the indium content at the four heights are given in the bottom row of Table I and show a significant increase towards the tip by almost a factor of 4 from positions (a)-(c).

Interestingly, for fitting the simulated line profile at position (d) (just below the tip) to the according experimental data, we had to assume a smaller indium content of only $18 \%$ compared to position (c). Hence, elastic deformation is reduced again after a certain height. However, previous studies found that a further increase of the indium content to the top is expected [52]. The origin of this decrease is found by STEM investigations (Sec. IIIB), showing a strong increase of plastic relaxation in the top region, most probably due to a higher indium content and, thus, a larger lattice mismatch which cannot be compensated for by purely elastic 
relaxation. Here, the crystal quality is significantly reduced compared to the bottom part of the rod.

Plastic relaxation is not included in the simulations and demands a critical interpretation of the obtained values for the indium content. However, if two mechanisms of strain relaxation are present-namely, plastic and elastic relaxation-our simulation, which considers only the elastic part, is expected to give a lower estimate of the indium content. Moreover, a clear trend of a significant gradient in the indium content towards the tip is observed in both approaches, and the values given in Table I are comparable to the values retrieved from STEM measurements (see Fig. 7).

In addition to nanofocus $x$-ray diffraction, we perform nanofocus x-ray-reflectivity measurements on the very same heights as sketched in Fig. 2. Along $q_{\text {rad }}$, we expect to reveal structural information about DQW and $\mathrm{GaN}$ barrier thickness. However, achieving this goal proves to be challenging, for several reasons. First, the intensity of the reflected signal decreases by more than 5 orders of magnitude within several degrees. Second, the decreased crystal quality due to defects in the top region of the rod leads to nonsharp crystal interfaces between (In, Ga)N and $\mathrm{GaN}$ regions. Moreover, the different parts of the rod, e.g., the shell, the barrier, and the DQW, are all inclined by several degrees, such that we observe up to three reflected streaks deviating by up to $5^{\circ}$ from the diffraction plane (not shown). However, in the angular direction, we are able to detect satellites referring to the facet width. Figure 5 shows experimentally retrieved nanofocus $x$-ray-reflectivity maps recorded at the very same positions: (a), (b), (c), and (d). In other words, the scattering plane for both diffraction and reflectivity remains the same and contains the facet normal, such that Fig. 2 applies here as well.

In addition to the main CTR, lateral satellites are visible in the $q_{\text {ang }}$ direction. The distance between subsequent minima along $q_{\text {ang }}$ refers to the width of the illuminated facet. As expected from the above-presented structural investigations of the rod, we observe a decrease of the spacing between the side oscillations translating to an increase of the facet width by about $200 \mathrm{~nm}$ from position (a) to position (d). Accordingly, the rod diameter increases from 1.3 to $1.7 \mu \mathrm{m}$. These values are comparable to SEM investigations of similar rods of the same sample (not shown) and enter the FEM simulations with the values given in red in Table I.

\section{B. Scanning transmission electron microscopy}

Using scanning transmission electron microscopy, we investigate several rods from the very same sample as the rod investigated by nanofocus $x$-ray diffraction in the previous section. Hence, the experimental results of both methods are comparable within the range of inhomogeneity of the sample. Using the FIB as outlined in Sec. II, a lamella containing several rods is isolated. The rods in the lamella are aligned in a way that an $m$ plane faces its neighbor's $m$ plane so that a projection onto the $\{11 \overline{2} 0\}$ lattice planes is possible. The lamella is further thinned by FIB to get rid of the inclined $m$ facets. Thus, in a cross-section STEM experiment, the electron beam is directed along the DQW in two opposing $m$ facets. The experiments are performed on a JEOL $2100 \mathrm{~F}$ microscope which is equipped with a scanning unit including a bright-field as well as a highangle annular dark-field (HAADF) detector. Using the latter, the experiments are mainly sensitive to the chemical composition of the sample (the $Z$ contrast).

Figure 6(a) displays a HAADF STEM cross-section micrograph of a representative individual rod. It shows that the GaN core thickness remains constant. However, the outer GaN shell thickness increases drastically. To quantify the thickness gradient, horizontal line profiles with an integration width of $100 \mathrm{~nm}$ of the HAADF intensity are taken at different rod heights. The inset in Fig. 6(b) shows that the thickness is obtained by measuring the distance between the peak originating from the outer quantum well and the turning point of the intensity profile. The intensity profile shown is taken at the height marked with the red arrow and results in the data point marked with a red box. Following this procedure, Fig. 6(b) shows the shell thickness as a function of rod height. It is clearly visible that the

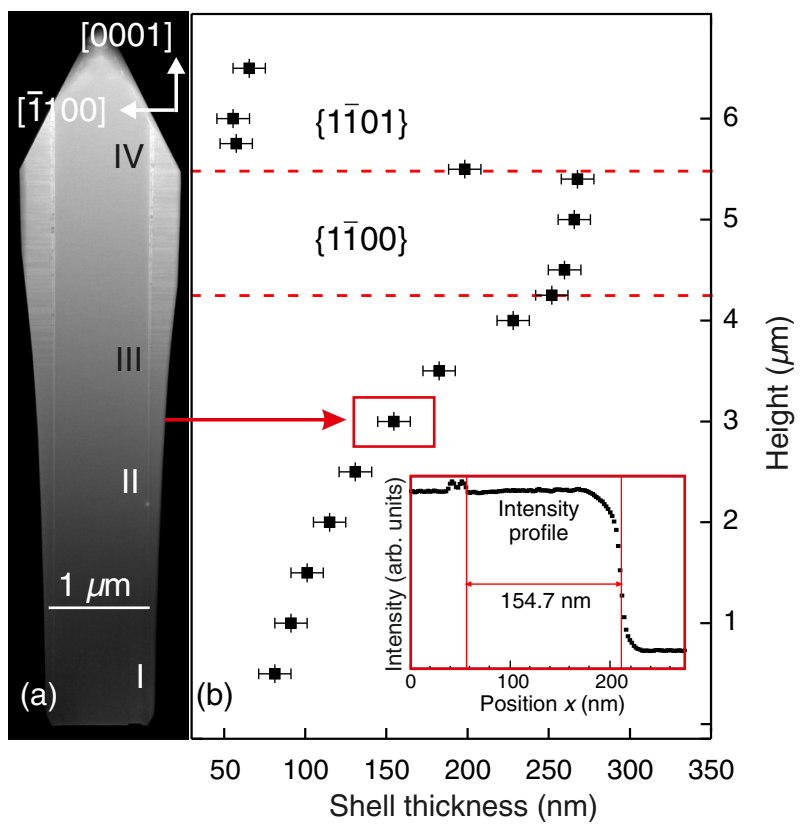

FIG. 6. (a) HAADF STEM cross-section micrograph of the $(\mathrm{In}, \mathrm{Ga}) \mathrm{N} / \mathrm{GaN}$ rod recorded along the [11 20$]$ direction. Positions I, II, III, and IV refer to a detailed STEM analysis (cf. Fig. 7). (b) Thickness of the outer GaN shell on the right side of the rod as a function of height. (Inset) An example showing how the intensity profile is used to determine the shell thickness. The presented case refers to a height indicated by the red arrow in (a), resulting in the data point marked with a red box in (b). Between the horizontal dashed red lines, the shell thickness approaches a constant value. 
shell thickness increases drastically from about 80 to about $270 \mathrm{~nm}$ from bottom to top. Interestingly, above approximately $4.2 \mu \mathrm{m}$, the shell thickness approaches a constant value and $m$ facets are actually formed. Owing to the formation of a tip with $\{1 \overline{1} 01\}$ facets, the shell thickness decreases above the second dashed line. The values fit very well to the shell thicknesses obtained from nanofocus $\mathrm{x}$ ray-diffraction measurements and substantiate the comparability of both results. Especially in the top region, both methods yield almost identical values. By comparing the shell thicknesses obtained with nanofocus x-ray diffraction with the values retrieved by STEM (see Fig. 6), it is possible to precisely correlate positions (a)-(d) to the actual heights. This correlation allows us to deduce the missing structural parameters (i.e., the DQW and barrier thicknesses) for the FEM simulations which could not be obtained from nanofocus x-ray-diffraction experiments.

At the positions I-IV marked in Fig. 6(a), HAADF STEM micrographs with higher magnification are taken in order to quantify the evolution of the quantum-well and barrier thicknesses as well as the indium content. The respective micrographs are shown in Fig. 7 (top panels). As the scale bar applies to all four micrographs, it is clearly visible that the DQW and the barrier increase in thickness. Moreover, at positions III and IV, stacking faults run horizontally from the DQW towards the GaN shell surface. Furthermore, at position IV, the crystal quality is significantly reduced.

Intensity line profiles with an integration width of about $180 \mathrm{~nm}$ are taken for positions I-IV and sigmoidal functions are used to fit the (In,Ga)N quantum-well intensity peaks to determine the FWHM, which is interpreted as the quantum-well thickness. The distance between the quantum-well peak positions subtracted by half of the width of each peak results in the $\mathrm{GaN}$ barrier thickness. The result is shown in Fig. 7 (bottom panel). Following the trend of the outer GaN shell, both the DQW and the barrier show a significant increase of their thickness from bottom (position I) to top (position IV). Up to approximately the middle of the rod, the quantum-well thickness remains constant at about $4 \mathrm{~nm}$. However, between positions III and IV, the thickness almost doubles. An even stronger increase in thickness is observed for the $\mathrm{GaN}$ barrier. Starting at about $1 \mathrm{~nm}$, the barrier thickness increases up to $16 \mathrm{~nm}$. The nanofocus $\mathrm{x}$-ray-diffraction experiment performed at the highest position [see Fig. 3(d)] shows broad oscillations along the CTRs, also referring to a thickness of $16 \mathrm{~nm}$. Hence, we can attribute these oscillations to the $\mathrm{GaN}$ barrier. Interestingly, despite the fact that the GaN barrier increases drastically, both the inner and outer quantum wells show very similar thicknesses over the full height.

In addition, the contrast of (In, Ga)N to GaN could be used to estimate the indium composition. For that reason, reference samples with known indium content are

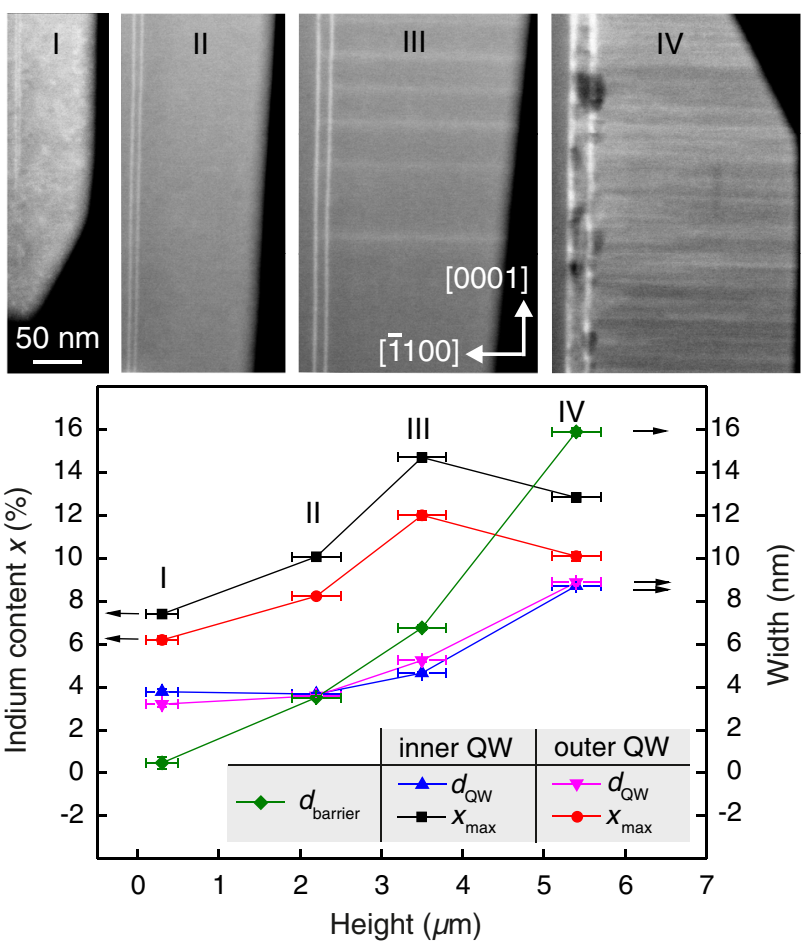

FIG. 7. (Top panels) HAADF STEM micrographs at the positions I-IV shown in Fig. 6. The scale bar applies to all four micrographs. At positions III and IV, stacking faults are visible which run horizontally from quantum wells (the vertical bright stripes) towards the GaN shell surface. By fitting the quantumwell peak intensity, the quantum-well thicknesses, $d_{\mathrm{QW}}$, the $\mathrm{GaN}$ barrier thickness, $d_{\text {barrier }}$, and the indium composition, $x_{\text {In }}$, could be extracted. Their evolution with respect to the rod height is shown in the bottom graph.

measured under exactly the same imaging conditions. Under the assumption $I \propto t Z^{\gamma}$, where $t$ is the thickness and $I$ is the HAADF intensity, a relationship between the contrast and the chemical composition described by the atomic number $Z$ could be deduced. A crucial parameter is $\gamma$, which describes the deviation from ideal Rutherford scattering [53]. The result in Fig. 7 (bottom panel) shows a significant increase along the rod height comparable to the result of the nanofocus x-ray-diffraction analysis. However, in the region where plastic relaxation sets in and voids are visible (position IV) the approach loses accuracy. Moreover, because of the superior spatial resolution of STEM compared to nanofocus x-ray diffraction, it is possible to resolve that the inner quantum well comprises an indium content that is up to $3 \%$ larger compared to the outer quantum well.

\section{SUMMARY}

In this paper, we investigate individual $(\mathrm{In}, \mathrm{Ga}) \mathrm{N} / \mathrm{GaN}$ core-shell microrods in terms of the structural properties, the local strain field, and the chemical composition via nanofocus x-ray diffraction and HAADF STEM. Thereby, 
we find that the multishell structure exhibits a significant increase in thickness in all subshells from bottom to top along the rod. Consequently, the rod diameter increases, and only at a certain height are $m$-plane facets formed. Based on the structural parameters retrieved from both methods, we perform finite-element method and subsequent kinematic scattering simulations. Owing to the fact that the structural parameters of the shells are well approximated, it is possible to fit the x-ray-diffraction profiles by the indium content, which is the sole free remaining parameter. In this manner, we find that the increase of shell thickness goes along with a significant increase in the indium content. As long as relaxation occurs purely elastically, this approach is expected to be reliable and is supported by a HAADF-STEM-based determination of the indium content yielding comparable values. Because of the higher resolution, HAADF STEM not only shows that the indium content increases but also that the indium content in the inner quantum well is higher than the outer one. Moreover, HAADF STEM measurements show that the top region of the rod is subject to plastic relaxation. Here, elastic relaxation decreases, and an increasing density of the horizontal partial-dislocation lines are observed. In the top area, an even higher indium content is expected; however, as only elastic relaxation is considered in the FEM simulations, only a lower limit of the actual indium content can be retrieved. The presented study shows that, by combining nanofocus x-ray diffraction complemented with FEM based kinematic scattering simulations and HAADF STEM on, ideally, the same object, deep insight on the structural and chemical constitution of lowdimensional semiconductors can be obtained.

\section{ACKNOWLEDGMENTS}

The authors would like to thank B. Jenichen for his critical reading of the manuscript, P. Alraun for the discussions, and A. Pfeiffer for the FIB preparation of the rods. Moreover, parts of this research are carried out within beam time I-20150179 at beam line P06 of the light source PETRAIII at DESY, a member of the Helmholtz Association (HGF). The financial support of the German Research Foundation, DFG (Projects No. HA3495/9-1 and No. FOR 1616), is gratefully acknowledged.

[1] B. Damilano, N. Grandjean, J. Massies, L. Siozade, and J. Leymarie, InGaN/GaN quantum wells grown by molecular-beam epitaxy emitting from blue to red at $300 \mathrm{~K}$, Appl. Phys. Lett. 77, 1268 (2000).

[2] T. Kuykendall, P. Ulrich, S. Aloni, and P. Yang, Complete composition tunability of InGaN nanowires using a combinatorial approach, Nat. Mater. 6, 951 (2007).

[3] T. R. Kuykendall, A. M. Schwartzberg, and S. Aloni, Gallium nitride nanowires and heterostructures: Toward color-tunable and white-light sources, Adv. Mater. 27, 5805 (2015).

[4] J. Johansson and K. A. Dick, Recent advances in semiconductor nanowire heterostructures, CrystEngComm 13, 7175 (2011).

[5] H. E. Jackson, L. M. Smith, and C. Jagadish, Recent advances in semiconductor nanowire heterostructures, ECS Trans. 64, 1 (2014).

[6] F. Qian, S. Gradečak, Y. Li, C.-Y. Wen, and C. M. Lieber, Core/multishell nanowire heterostructures as multicolor, high-efficiency light-emitting diodes, Nano Lett. 5, 2287 (2005).

[7] H.-W. Lin, Y.-J. Lu, H.-Y. Chen, H.-M. Lee, and S. Gwo, InGaN/GaN nanorod array white light-emitting diode, Appl. Phys. Lett. 97, 073101 (2010).

[8] W. Guo, A. Banerjee, P. Bhattacharya, and B. S. Ooi, InGaN/GaN disk-in-nanowire white light emitting diodes on (001) silicon, Appl. Phys. Lett. 98, 193102 (2011).

[9] F. Limbach, C. Hauswald, J. Lähnemann, M. Wölz, O. Brandt, A. Trampert, M. Hanke, U. Jahn, R. Calarco, L. Geelhaar, and H. Riechert, Current path in light emitting diodes based on nanowire ensembles, Nanotechnology 23, 465301 (2012).

[10] M. Mandl, X. Wang, T. Schimpke, C. Kölper, M. Binder, J. Ledig, A. Waag, X. Kong, A. Trampert, F. Bertram, J. Christen, F. Barbagini, E. Calleja, and M. Strassburg, Group III nitride core-shell nano- and microrods for optoelectronic applications, Phys. Status Solidi RRL 7, 800 (2013).

[11] M. Mikulics, A. Winden, M. Marso, A. Moonshiram, H. Lüth, D. Grützmacher, and H. Hardtdegen, Nano-lightemitting-diodes based on InGaN mesoscopic structures for energy saving optoelectronics, Appl. Phys. Lett. 109, 041103 (2016).

[12] A. Waag, X. Wang, S. Fündling, J. Ledig, M. Erenburg, R. Neumann, M. Al Suleiman, S. Merzsch, J. Wei, S. Li, H. H. Wehmann, W. Bergbauer, M. Strassburg, A. Trampert, U. Jahn, and H. Riechert, The nanorod approach: GaN nanoLEDs for solid state lighting, Phys. Status Solidi (a) 8, 2296 (2011).

[13] S. Li and A. Waag, GaN based nanorods for solid state lighting, J. Appl. Phys. 111, 071101 (2012).

[14] P. Horenburg, E. R. Buß, U. Rossow, H. Bremers, F. A. Ketzer, and A. Hangleiter, Strain dependence of In incorporation in $m$-oriented GaInN/GaN multi quantum well structures, Appl. Phys. Lett. 108, 102105 (2016).

[15] T. Schimpke, M. Mandl, I. Stoll, B. Pohl-Klein, D. Bichler, F. Zwaschka, J. Strube-Knyrim, B. Huckenbeck, B. Max, M. Müller, P. Veit, F. Bertram, J. Christen, J. Hartmann, A. Waag, H.-J. Lugauer, and M. Strassburg, Phosphorconverted white light from blue-emitting InGaN microrod LEDs, Phys. Status Solidi (a) 213, 1577 (2016).

[16] V. Favre-Nicolin, F. Mastropietro, J. Eymery, D. Camacho, Y. M. Niquet, B. M. Borg, M. E. Messing, L.-E. Wernersson, R. E. Algra, E. P. A. M. Bakkers, T. H. Metzger, R. Harder, and I. K. Robinson, Analysis of strain and stacking faults in single nanowires using Bragg coherent diffraction imaging, New J. Phys. 12, 035013 (2010).

[17] F. Glas, J.-C. Harmand, and G. Patriarche, Why Does Wurtzite Form in Nanowires of III-V Zinc Blende Semiconductors?, Phys. Rev. Lett. 99, 146101 (2007). 
[18] J. Eymery, F. Rieutord, V. Favre-Nicolin, O. Robach, Y.-M. Niquet, L. Fröberg, T. Mårtensson, and L. Samuelson, Strain and shape of epitaxial InAs/InP nanowire superlattice measured by grazing incidence $\mathrm{x}$-ray techniques, Nano Lett. 7, 2596 (2007).

[19] A. Biermanns, D. Carbone, S. Breuer, V. L. R. Jacques, T. Schulli, L. Geelhaar, and U. Pietsch, Distribution of zincblende twins and wurtzite segments in GaAs nanowires probed by x-ray nanodiffraction, Phys. Status Solidi RRL 7, 860 (2013).

[20] M. Keplinger, T. Mårtensson, J. Stangl, E. Wintersberger, B. Mandl, D. Kriegner, V. Holý, G. Bauer, K. Deppert, and L. Samuelson, Structural investigations of core-shell nanowires using grazing incidence $\mathrm{x}$-ray diffraction, Nano Lett. 9, 1877 (2009).

[21] G. Tourbot, C. Bougerol, A. Grenier, M. Den Hertog, D. Sam-Giao, D. Cooper, P. Gilet, B. Gayral, and B. Daudin, Structural and optical properties of InGaN/GaN nanowire heterostructures grown by PA-MBE, Nanotechnology 22, 075601 (2011).

[22] G. Tourbot, C. Bougerol, F. Glas, L. F. Zagonel, Z. Mahfoud, S. Meuret, P. Gilet, M. Kociak, B. Gayral, and B. Daudin, Growth mechanism and properties of $\mathrm{InGaN}$ insertions in GaN nanowires, Nanotechnology 23, 135703 (2012).

[23] M. Heurlin, T. Stankevič, S. Mickevičius, S. Yngman, D. Lindgren, A. Mikkelsen, R. Feidenhans'1, M. T. Borgström, and L. Samuelson, Structural properties of wurtzite InPInGaAs nanowire core-shell heterostructures, Nano Lett. 15, 2462 (2015).

[24] J. Segura-Ruiz, G. Martínez-Criado, J. A. Sans, R. Tucoulou, P. Cloetens, I. Snigireva, C. Denker, J. Malindretos, A. Rizzi, M. Gomez-Gomez, N. Garro, and A. Cantarero, Direct observation of elemental segregation in $\mathrm{InGaN}$ nanowires by X-ray nanoprobe, Phys. Status Solidi RRL 5, 95 (2011).

[25] J. Segura-Ruiz, G. Martínez-Criado, C. Denker, J. Malindretos, and A. Rizzi, Phase separation in single $\mathrm{In}_{x} \mathrm{Ga}_{1-x} \mathrm{~N}$ nanowires revealed through a hard x-ray synchrotron nanoprobe, Nano Lett. 14, 1300 (2014).

[26] M. K. Horton, S. Rhode, S.-L. Sahonta, M. J. Kappers, S. J. Haigh, T. J. Pennycook, C. J. Humphreys, R. O. Dusane, and M. A. Moram, Segregation of In to dislocations in InGaN, Nano Lett. 15, 923 (2015).

[27] M. Niehle, A. Trampert, S. Albert, A. Bengoechea-Encabo, and E. Calleja, Electron tomography of (In, Ga)N insertions in GaN nanocolumns grown on semi-polar (112̄2) GaN templates, APL Mater. 3, 036102 (2015).

[28] M. Hanke, C. Eisenschmidt, P. Werner, N. D. Zakharov, F. Syrowatka, F. Heyroth, P. Schäfer, and O. Konovalov, Elastic strain relaxation in axial $\mathrm{Si} / \mathrm{Ge}$ whisker heterostructures, Phys. Rev. B 75, 161303 (2007).

[29] S. Conesa-Boj, F. Boioli, E. Russo-Averchi, S. Dunand, M. Heiss, D. Rüffer, N. Wyrsch, C. Ballif, L. Miglio, and A. F. I. Morral, Plastic and elastic strain fields in GaAs/Si core-shell nanowires, Nano Lett. 14, 1859 (2014).

[30] T. Etzelstorfer, M. J. Süess, G. L. Schiefler, V. L. R. Jacques, D. Carbone, D. Chrastina, G. Isella, R. Spolenak, J. Stangl, H. Sigg, and A. Diaz, Scanning x-ray strain microscopy of inhomogeneously strained Ge micro-bridges, J. Synchrotron Radiat. 21, 111 (2014).
[31] M. Wölz, M. Ramsteiner, V. M. Kaganer, O. Brandt, L. Geelhaar, and H. Riechert, Strain engineering of nanowire multi-quantum well demonstrated by Raman spectroscopy, Nano Lett. 13, 4053 (2013).

[32] J. Kioseoglou, T. Pavloudis, T. Kehagias, P. Komninou, T. Karakostas, C. D. Latham, M. J. Rayson, P. R. Briddon, and M. Eickhoff, Structural and electronic properties of GaN nanowires with embedded $\mathrm{In}_{x} \mathrm{Ga}_{1-x} \mathrm{~N}$ nanodisks, J. Appl. Phys. 118, 034301 (2015).

[33] I. Robinson and R. Harder, Coherent x-ray diffraction imaging of strain at the nanoscale, Nat. Mater. 8, 291 (2009).

[34] J. Stangl, C. Mocuta, V. Chamard, and D. Carbone, Nanobeam X-Ray Scattering (Wiley-VCH, Weinheim, 2013).

[35] M. Keplinger, B. Mandl, D. Kriegner, V. Holý, L. Samuelsson, G. Bauer, K. Deppert, and J. Stangl, X-ray diffraction strain analysis of a single axial $\operatorname{InAs}_{1-x} \mathrm{P}_{x}$ nanowire segment, J. Synchrotron Radiat. 22, 59 (2015).

[36] M. Keplinger, R. Grifone, J. Greil, D. Kriegner, J. Persson, A. Lugstein, T. Schülli, and J. Stangl, Strain distribution in single, suspended germanium nanowires studied using nanofocused X-rays, Nanotechnology 27, 055705 (2016).

[37] D. Dzhigaev, A. Shabalin, T. Stankevič, U. Lorenz, R. P. Kurta, F. Seiboth, J. Wallentin, A. Singer, S. Lazarev, O. M. Yefanov, M. Borgström, M. N. Strikhanov, L. Samuelson, G. Falkenberg, C. G. Schroer, A. Mikkelsen, R. Feidenhans'1, and I. A. Vartanyants, Bragg coherent x-ray diffractive imaging of a single indium phosphide nanowire, J. Opt. 18, 064007 (2016).

[38] M. Hanke, M. Dubslaff, M. Schmidbauer, T. Boeck, S. Schröder, M. Burghammer, C. Riekel, J. Patommel, and C. G. Schroer, Scanning x-ray diffraction with $200 \mathrm{~nm}$ spatial resolution, Appl. Phys. Lett. 92, 193109 (2008).

[39] M. Dubslaff, M. Hanke, M. Burghammer, S. Schröder, R. Hoppe, C. G. Schroer, Y. I. Mazur, Z. M. Wang, J. H. Lee, and G. J. Salamo, In(Ga)As/GaAs(001) quantum dot molecules probed by nanofocus high resolution $\mathrm{x}$-ray diffraction with $100 \mathrm{~nm}$ resolution, Appl. Phys. Lett. 98, 213105 (2011).

[40] J. Gulden, S. O. Mariager, A. P. Mancuso, O. M. Yefanov, J. Baltser, P. Krogstrup, J. Patommel, M. Burghammer, R. Feidenhans'1, and I. A. Vartanyants, Coherent x-ray nanodiffraction on single GaAs nanowires, Phys. Status Solidi (a) 208, 2495 (2011).

[41] T. Krause, M. Hanke, Z. Cheng, M. Niehle, A. Trampert, M. Rosenthal, M. Burghammer, J. Ledig, J. Hartmann, H. Zhou, H.-H. Wehmann, and A. Waag, Nanofocus x-ray diffraction and cathodoluminescence investigations into individual core-shell ( $\mathrm{In}, \mathrm{Ga}) \mathrm{N} / \mathrm{GaN}$ rod light-emitting diodes, Nanotechnology 27, 325707 (2016).

[42] T. Stankevič, D. Dzhigaev, Z. Bi, M. Rose, A. Shabalin, J. Reinhardt, A. Mikkelsen, L. Samuelson, G. Falkenberg, I. A. Vartanyants, and R. Feidenhans'l, Strain mapping in an InGaN/GaN nanowire using a nano-focused x-ray beam, Appl. Phys. Lett. 107, 103101 (2015).

[43] T. Stankevič, E. Hilner, F. Seiboth, R. Ciechonski, G. Vescovi, O. Kryliouk, U. Johansson, L. Samuelson, G. Wellenreuther, G. Falkenberg, R. Feidenhans'1, and A. Mikkelsen, Fast strain mapping of nanowire light-emitting 
diodes using nanofocused x-ray beams, ACS Nano 9, 6978 (2015).

[44] C. Tessarek, M. Heilmann, E. Butzen, A. Haab, H. Hardtdegen, C. Dieker, E. Spiecker, and S. Christiansen, The role of $\mathrm{Si}$ during the growth of $\mathrm{GaN}$ micro- and nanorods, Cryst. Growth Des. 14, 1486 (2014).

[45] J. Hartmann, X. Wang, H. Schuhmann, W. Dziony, L. Caccamo, J. Ledig, M.S. Mohajerani, T. Schimpke, M. Bähr, G. Lilienkamp, W. Daum, M. Seibt, M. Strassburg, H.-H. Wehmann, and A. Waag, Growth mechanisms of GaN microrods for 3D core-shell LEDs: The influence of silane flow, Phys. Status Solidi (a) 212, 2830 (2015).

[46] M. Niehle and A. Trampert, Electron tomography on nanopores embedded in epitaxial GaSb thin films, Micron 73, 54 (2015).

[47] C. G. Schroer, P. Boye, J. M. Feldkamp, J. Patommel, D. Samberg, A. Schropp, A. Schwab, S. Stephan, G. Falkenberg, G. Wellenreuther, and N. Reimers, Hard X-ray nanoprobe at beamline P06 at PETRA III, Nucl. Instrum. Methods Phys. Res., Sect. A 616, 93 (2010).

[48] C. G. Schroer, M. Kuhlmann, U. T. Hunger, T. F. Günzler, O. Kurapova, S. Feste, F. Frehse, B. Lengeler, M. Drakopoulos,
A. Somogyi, A. S. Simionovici, A. Snigirev, I. Snigireva, C. Schug, and W. H. Schröder, Nanofocusing parabolic refractive X-ray lenses, Appl. Phys. Lett. 82, 1485 (2003).

[49] C. G. Schroer, O. Kurapova, J. Patommel, P. Boye, J. Feldkamp, B. Lengeler, M. Burghammer, C. Riekel, L. Vincze, A. van der Hart, and M. Küchler, Hard X-ray nanoprobe based on refractive x-ray lenses, Appl. Phys. Lett. 87, 124103 (2005).

[50] M. Hanke, M. Schmidbauer, and R. Köhler, Lateral correlation of $\mathrm{SiGe}$ Stranski-Krastanow islands on silicon as probed by high resolution x-ray diffraction, J. Appl. Phys. 96, 1959 (2004).

[51] K. Shimada, First-principles determination of piezoelectric stress and strain constants of wurtzite III-V nitrides, Jpn. J. Appl. Phys. 45, L358 (2006).

[52] J. Ledig, X. Wang, S. Fündling, H. Schuhmann, M. Seibt, U. Jahn, H.-H. Wehmann, and A. Waag, Characterization of the internal properties of $\mathrm{InGaN} / \mathrm{GaN}$ core-shell LEDs, Phys. Status Solidi (a) 213, 11 (2016).

[53] P. Hartel, H. Rose, and C. Dinges, Conditions and reasons for incoherent imaging in STEM, Ultramicroscopy 63, 93 (1996). 